\title{
Mensurações em ciência
}

Measurements in science

Harley Edson Amaral Bicas 1

${ }^{1}$ Professor Titular, Departamento de Oftalmologia, Otorrinolaringologia e Cirurgia de Cabeça e Pescoço, Faculdade de Medicina de Ribeirão Preto da Universidade de São Paulo - USP.

Endereço para correspondência: Depto. OFT/ORL/ CCP, Av. Bandeirantes, 3900 - Ribeirão Preto (SP) CEP 14019-900

INTRODUÇÃO

Embora se saiba que o conhecimento humano não tem, em todo o seu espectro, a dependência restrita do que seja mensurável, toma-se a quantificação como o requisito básico da ciência, pelo menos as naturais. E ainda que hoje possa prevalecer, inclusive nelas, uma certa desconfiança em torno do célebre aforisma positivista do século passado, "o que não pode ser medido, não é assunto científico", ele continua essencialmente válido, não se admitindo que o método científico dispense a possibilidade de contagens e cálculos. Sendo então as mensurações tão essenciais ao método científico natural, convém uma revisão sobre alguns de seus aspectos principais.

\section{A) Sistemas numéricos}

A representações de quantidades dão-se "nomes" (por exemplo, os das contagens um, dois, três, sete, nove) e "símbolos" ou algarismos (respectivamente 1, 2, 3, 7, 9). Embora não haja limite para a quantidade desses nomes e símbolos, fica muito pouco prático se ela for muito grande. A retomada deles, em novas séries, pode então facilitar contagens maiores. Isto é, a quantificação pode continuar ascendente, sem empregar algarismos maiores, apenas reutilizando os já existentes em associações progressivas. Para isso, usa-se o "zero", o conceito de algarismo pelo qual, num sistema de quantificação, pode-se "ascender" de uma série a outra. O sistema métrico moderno é o decimal, composto por dez algarismos (um a nove e mais o zero). Com o zero passa-se da série dessas unidades à das dezenas, depois à das centenas, em seguida à dos milhares e assim sucessivamente.

Um sistema operacionalmente melhor é o duodecimal (onze algarismos e mais o zero), pelo qual sua base (12) pode ser "perfeitamente" dividida por quatro de seus algarismos constituintes $(2,3,4$ e 6$)$ enquanto a base do decimal (10) só pode ser dividida por dois deles (o 2 e o 5). Mas o mais simples dos sistemas de contagem é o binário que conta com apenas dois números: o um e o zero (o sim e o não, o "ligado" e o "desligado", o "branco" e o "preto", o "sinal" e o "silêncio"), a linguagem aritmética usada pelos computadores e instrumentos digitais (de "dígitos", números). 


\section{B) Representações logarítmicas}

Em qualquer sistema operacional (binário, decimal, duodecimal, etc.) um número $(\mathrm{N})$ pode ser representado por uma notação exponencial (p) da respectiva base (B), isto é, $N=B^{p}$. No sistema decimal, por exemplo:

$$
\begin{array}{rlr}
10^{0} & = & 1 \\
10^{1} & = & 10 \\
10^{2} & = & 100 \\
10^{3} & = & 1000 \\
10^{4} & = & 10000
\end{array}
$$

Note-se que o expoente da base (10) corresponde ao número adicional de "casas" além da série unitária básica (1...9). Obviamente, pode-se ter um expoente fracionário, cuja correspondência seja a de um número "inteiro", ou fracionário:

$$
\begin{array}{ll}
10^{1,491361694} & =31 \\
10^{1,5} & =31,6227766 \\
10^{1,505149978} & =32
\end{array}
$$

Diz-se, então, que "o logaritmo decimal (ou na base dez) de 31 é 1,491361694". Em outras palavras: logaritmo de um número (N) é o expoente (p) ao qual a base do sistema (B) deve ser elevada para dar aquele número. Assim, também, $2^{8}=256$, significa que o logaritmo de 256 no sistema binário é 8 .

Note-se que $10^{0,491361694}=3,1$ enquanto $10^{2,491361694}=310$. À parte "inteira" do expoente (isto é, do logaritmo) dá-se o nome "característica" (do logaritmo) e à parte fracionária (e.g., 0,491361694), “mantissa".

Claro, também podem ocorrer logaritmos negativos (ou seja, frações):

$$
\begin{aligned}
10^{\circ} & =1 \\
10^{-1} & =0,1 \\
10^{-2} & =0,01 \\
10^{-3} & =0,001
\end{aligned}
$$

E, então:

$$
10^{(0,491361694-1)}=10^{-0,508638306}=0,31
$$

Uma outra representação logarítmica muito usada na ciência é a dos logaritmos naturais ou neperianos. Nela, a base é $\mathrm{e}=2,718281828$.

\section{C) Unidades. O Sistema Internacional de medidas.}

Em certos casos, a contagem com que se expressa uma quantidade é feita em unidades "naturais", em números "inteiros" e individualizados. Por exemplo, a quantidade de alunos numa sala é 37 (isto é, alunos). O "indivíduo" é contado como unidade (número). Diz-se também: o número de alunos na sala é 37. O número de gatos na ninhada é 6 . Há 6 gatos na ninhada.

Em outros casos, por exemplo, para medida da distância entre dois pontos, duas cidades, etc. não há uma unidade "natural" e óbvia. Mas pode-se arbitrar uma quantidade de distância, tomá-la como unidade (de distância) e fazer dela uma referência, sobre a qual outras medidas (de distância) possam ser avaliadas. Assim, por exemplo, para medir comprimentos pode falar-se em "metros", ou em "jardas", ou "pés", que são convenções estabelecidas. Unidades de massa são o "quilograma", a "libra", a "onça", etc.

Obviamente, enorme confusão existiria se cada pessoa, ou país, adotasse uma determinada unidade para fazer as suas medidas. Assim, convencionou-se internacionalmente que as unidades, embora possam ser arbitrariamente criadas, deveriam ser padronizadas, para formar um sistema unificado e coerente de medidas, que chegasse a ser entendido em qualquer país e por qualquer pessoa. Estabeleceu-se então um Sistema Internacional (S.I.) de medidas, pelo qual as unidades básicas de grandeza fiquem rigidamente padronizadas e definidas de modo que, a partir delas, as quantificações sejam expressas. Assim, por exemplo, a unidade de comprimento (distância) no S.I. é o metro. Desde 1889 a 1960 prevaleceu uma definição baseada no protótipo internacional desse valor, à distância de dois traços em uma barra de platina iridiada. Em 1960 esse padrão foi substituído por outro (baseado no comprimento de onda de uma radiação do criptônio 86), mas desde a 17a Conferência Geral de Pesos e Medidas, em 1983, prevalece a de que "O metro (m) é o comprimento do trajeto percorrido pela luz no vácuo durante um intervalo de tempo de 1/299.792.458 de segundo (17 a CGPM, 1963, resolução 1)".

Certamente uma definição complicada (e que leva em conta o valor de uma outra variável, o tempo), mas que constitui o padrão científico inequívoco para medidas de distância. Ora, em certos países ainda são usadas unidades obsoletas como a milha, a jarda, o pé, a polegada, cujos valores devem ser evitados no uso científico e convertidos aos da unidade métrica do S.I. Por exemplo:

$$
\begin{aligned}
1 \mathrm{mi}(\text { milha }) & =1609,3440 \mathrm{~m} \\
1 \mathrm{yd}(\text { jarda }) & =0,9144 \mathrm{~m} \\
1 \mathrm{ft}(\text { pé }) & =0,3048 \mathrm{~m} \\
1 \mathrm{in}(\text { polegada }) & =0,0254 \mathrm{~m}
\end{aligned}
$$

A unidade de massa é o quilograma, $\mathrm{kg}$ (igual à massa do protótipo internacional do quilograma, sancionado pela $1^{\text {a }}$ CGPM, em 1889 e conservado até hoje), a do tempo é o segundo, s (duração de 9.192.631.770 períodos da radiação correspondente à transição entre os dois níveis hiperfinos do estado fundamental do átomo de Césio 133, conforme decidido na 13a CGPM, 1967, Resolução 1), a de corrente elétrica é o ampére (A), a de temperatura termodinâmica o kelvin (K), a de quantidade de matéria o mol (mol), a de intensidade luminosa a candela (cd), todas elas chamadas "unidades de base" e das quais se originam as "derivadas" (por exemplo, a de superfície, $\mathrm{m}^{2}$; a de velocidade, $\mathrm{m} / \mathrm{s}$, a de luminância, $\mathrm{cd} / \mathrm{m}^{2}$, etc.).

Em algumas situações, há uma relação entre duas unidades de mesma grandeza, de que, por simplificação, resulta um número puro, isto é, sem unidades. Há alguns exemplos disso, em Oftalmologia.

\section{1) Índice de refração (n)}

É um valor que exprime a relação entre a velocidade da luz encontrada no material (c) cujo $\mathbf{n}$ se quer medir e a no vácuo $\left(c_{\mathrm{o}}=299.792 .458 \mathrm{~m} / \mathrm{s}\right)$ : 


$$
\mathrm{n}=\frac{\mathrm{c}_{\mathrm{o}}}{\mathrm{c}} \frac{\mathrm{m} / \mathrm{s}}{\mathrm{m} / \mathrm{s}}
$$

Por exemplo, para o acrílico, c = 201.202.992 m/s. Então:

$$
\mathrm{n}=\frac{299.792 .458 \mathrm{~m} / \mathrm{s}}{201.202 .992 \mathrm{~m} / \mathrm{s}}=1,49
$$

\section{2) Acuidade visual}

A acuidade visual (AV) normal, ou padrão, ou unitária (AV= $1,0)$ é definida como a recíproca (inverso) do ângulo plano de um minuto de arco. No S.I., os ângulos planos são considerados como grandezas com uma das unidades chamadas suplementares, o radiano (rad). Há $2 \pi \mathrm{rad}$ em uma circunferência $\left(360^{\circ}=21600^{\prime}\right)$, de modo que um minuto de arco $\left(1^{\prime}\right)$ é aproximadamente igual a $0,000291 \mathrm{rad}$. O inverso desse valor, ou seja $1 / 1^{\prime}=1 \div(2 \pi / 21600)$ é $10800 / \pi=3437,746771 \mathrm{rad}^{-1}$, corresponde pois, teoricamente, ao que seria a unidade de acuidade visual no Sistema Internacional. Uma $\mathrm{AV}=0,2$ (equivalente ao ângulo de 5 minutos de arco) seria então dada por $3437,747 \times 0,2=$ $687,549 \mathrm{rad}^{-1}$. Não há um nome específico para essa unidade. Por outro lado, os valores trigonométricos (seno, cosseno, tangente) de ângulos muito pequenos (como os de minutos de arco) podem ser convenientemente expressos pela relação de comprimentos (lados) de um triângulo, ou seja, por exemplo (no triângulo $\mathrm{ABC}$ da figura 1 ): $\tan \mathrm{a}=\overline{\mathrm{AB}} / \overline{\mathrm{AC}} \simeq \mathrm{a}$.

E então, como tan $1^{\prime} \simeq 0,000291 \simeq$ a (note-se a "coincidência" numérica com o valor de $1^{\prime}$ em radianos), vem $1 \mathrm{a}^{-1}=$ 3437,746674 , um número puro, sem unidades (e praticamente idêntico ao já dado anteriormente como "unidade de AV" no S.I.). Mas por sua vez, esse valor "unitário" ( $\left.1 \mathrm{a}^{-1}\right)$ assim definido, é equivalente à relação $\overline{\mathrm{AC}} / \overline{\mathrm{AB}}$. Sendo $\overline{\mathrm{AC}}=\mathrm{d}$ a distância do olho ao optotipo e $\overline{\mathrm{AB}}=\mathrm{h}$ o tamanho deste:

$$
\mathrm{a}^{-1}=\mathrm{d} / \mathrm{h}
$$

Costuma-se, também, exprimir um valor de "acuidade visual" como a relação entre a recíproca de dois valores angulares $\left(\mathrm{a}^{-1}\right.$ e $\left.\mathrm{a}_{1}^{-1}\right)$, isto é:

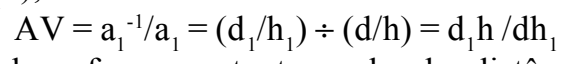

Ora, pode-se fazer constante o valor das distâncias em que as medidas são feitas $\left(\mathrm{d}=\mathrm{d}_{1}\right)$ de modo que a AV apareça como expressão da relação de tamanhos de optotipos: o de tamanho padronizado como normal para aquela distância (h) e o que é nela percebido $\left(\mathrm{h}_{1}\right)$ :

$$
\mathrm{AV}=\mathrm{h} / \mathrm{h}_{1}
$$

Por outro lado, pode-se fixar o tamanho do optotipo $(\mathrm{h}=$ $h_{1}$ ) e medir a distância em que ele se torna percebido, isto é:

$$
\mathrm{AV}=\mathrm{d}_{1} / \mathrm{d}
$$

Curiosamente, usa-se ainda uma outra referência: fixa-se a distância d em que se faz a avaliação (como no primeiro caso) mas menciona-se a medida da AV como se fosse uma relação de distâncias, isto é, como se elas variassem. A relação passa a ser entre a da distância de teste (d) e a daquela em que o menor optotipo discriminado seria normalmente visível $\left(\mathrm{d}_{2}\right)$. Essa transformação tem a seguinte base: o optotipo, cujo tamanho $\left(\mathrm{h}_{2}\right)$ à distância padrão de teste $(\mathrm{d})$ corresponde a um ângulo $\mathrm{a}_{2}$, seria normalmente visível à distância $\mathrm{d}_{2}$, tal que a (ângulo visual normal, padrão) $=\mathrm{h}_{2} / \mathrm{d}_{2}$. Portanto:

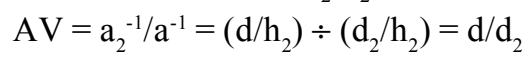

Note-se que toda essa "ginástica" faz com que a distância em que é feito o teste (d) passe do denominador (quando a AV é medida com tamanho constante do optotipo) ao numerador, dando-se a fração como se fosse eqüivalente à relação de tamanhos de optotipos à distância constante $\left(\mathrm{h} / \mathrm{h}_{1}\right)$. Tal expressão é a que se usa nas notações de acuidade visual dos países anglo-saxônicos, em que o valor de d (distância em que se faz a avaliação) aparece como 20 pés e o de $d_{2}$ como (por exemplo) 400 pés, de modo que, então, AV = 20/400. Obviamente, a expressão decimal $(\mathrm{AV}=0,05)$ é equivalente. Claro que a notação fracionária poderá também ser usada. Mas, então, mais apropriadamente, na medida internacionalmente convencionada para distâncias, o metro: $\mathrm{AV}=6 / 6^{(*)}$, principalmente em países nos quais não se usa a medida métrica "pés". De qualquer modo, como relações de dimensões de mesma natureza $\left(\mathrm{h} / \mathrm{h}_{1}\right.$ ou $\mathrm{d}_{1} / \mathrm{d}$ ou d$\left./ \mathrm{d}_{2}\right)$, a acuidade visual pode, também, ser considerada como uma grandeza sem unidades, um número "puro".

\section{3) Dioptria-prismática}

Aqui, novamente, a ambigüidade entre "unidade" ou "sem unidade" para a expressão de uma medida angular volta a ocorrer. Por definição, "dioptria-prismática" é o nome dado à relação de duas dimensões métricas, significando um ângulo. Uma dioptria-prismática é a dimensão do ângulo formado pela separação de $1 \mathrm{~cm}$ entre dois pontos, a um metro de distância (quando, na figura $1, \mathrm{AB}=1 \mathrm{~cm} ; \mathrm{AC}=100 \mathrm{~cm}$ ).

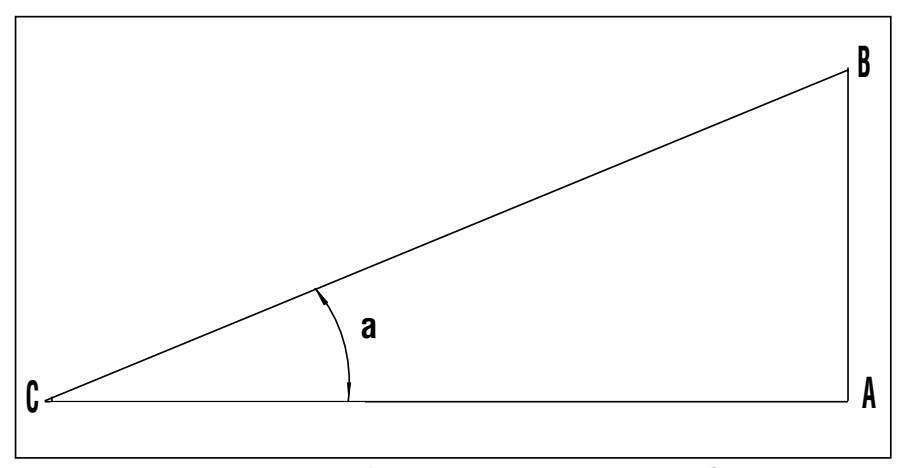

Figura 1. Quantificação de ângulos em dioptrias-prismáticas (P). Por definição $P=\overline{A B} / \overline{A C}$, quando $\overline{A B}$ é expresso em centímetros e $\overline{A C}$ em metros. $\log 0 \mathrm{P}=100 \overline{\mathrm{AB}} / \overline{\mathrm{AC}}$.

Ora, tal relação, portanto, não tem unidades (não tem dimensão), ainda que receba uma denominação (dioptria-prismática). Por outro lado, exprimindo medida de ângulo plano,

\footnotetext{
(*) Na verdade 20 pés equivalem a $6,096 \mathrm{~m}$ ou, reciprocamente, 6 metros representam 19,685 pés.
} 
essa "dimensão" pode ser dada como recíproca do radiano. De fato, $\mathrm{P}=100 \overline{\mathrm{AB}} / \overline{\mathrm{AC}}$ e tan $\mathrm{a}=\overline{\mathrm{AB}} / \overline{\mathrm{AC}}$. Portanto $\mathrm{P}=100$ tan $\mathrm{a}$. Então, para o valor de uma dioptria prismática o ângulo é $0,572938697^{\circ}=0,999966668.10^{-2} \mathrm{rad} \simeq 1$ centrad. Essa equivalência prática entre o valor da dioptria-prismática e o centésimo do radiano (subunidade à qual se deu o nome centrad) suscita a discussão de se a dioptria-prismática deva ser relacionada ao ângulo (que quer efetivamente medir) ou à sua tangente (como vem de sua definição). Num caso $P=f(a)$, a relação é constante. No outro, $\mathrm{P}=\mathrm{f}(\tan \mathrm{a})$, é progressivamente crescente. A diferença pode ser entendida pela figura 2.

Note-se que:

Mas

$\tan \mathrm{a}=\overline{\mathrm{AB}} / \overline{\mathrm{AC}}=\overline{\mathrm{DE}} / \overline{\mathrm{DC}}=\overline{\mathrm{GH}} / \overline{\mathrm{GC}}=\overline{\mathrm{JK}} / \overline{\mathrm{JC}}$ $\tan 2 \mathrm{a}=\overline{\mathrm{AF}} / \overline{\mathrm{AC}}=(\overline{\mathrm{AB}}+\overline{\mathrm{BF}}) / \overline{\mathrm{AC}}=$ $(\tan \mathrm{a})+(\overline{\mathrm{BF}} / \overline{\mathrm{AC}})$

E como

$\overline{\mathrm{BF}}>\overline{\mathrm{DE}}=\overline{\mathrm{AB}}$, então $\overline{\mathrm{BF}} / \overline{\mathrm{AC}}>\tan \mathrm{a}$

Portanto

Assim, também,

$$
\tan 2 \mathrm{a}>2 \text { tan } \mathrm{a}
$$

$\tan (\overline{\mathrm{AI}} / \overline{\mathrm{AC}})=\tan 3 \mathrm{a}>3 \tan \mathrm{a}$, $\tan (\overline{\mathrm{AL}} / \overline{\mathrm{AC}})=\tan 4 \mathrm{a}>4$ tan $\mathrm{a}$, etc.

\section{D) Ordens de grandeza}

Quando exprimindo um número muito grande ou muito pequeno (fracionário) a notação extensa de uma grandeza torna-se inadequada, usando-se para substitui-la a notação exponencial. Por exemplo, em 500 dias a luz percorre uma distância aproximada de $(500 \times 24$ × 60 × 60) segundos $\times$ $300.000 .000 \mathrm{~m} / \mathrm{s}=12.960 .000 .000 .000 .000 \mathrm{~m}$, uma quantidade de expressão desconfortante (extensa) e de uso delicado (o número de casas pode confundir). Melhor então escrevê-la como $1296.10^{13} \mathrm{~m}$, em que o expoente (13) representa o número de "casas" (zeros) ou, ainda, 1,296.10 ${ }^{16} \mathrm{~m}$. Assim, também, o comprimento de onda de uma luz vermelha medido como $0,0000007 \mathrm{~m}$, é mais facilmente anotado como $7 \cdot 10^{-7} \mathrm{~m}$.

Os valores exponenciais para representar múltiplos ou submúltiplos da unidade recebem, então, nomes especiais, compostos por prefixos a serem usados com a unidade, confe-

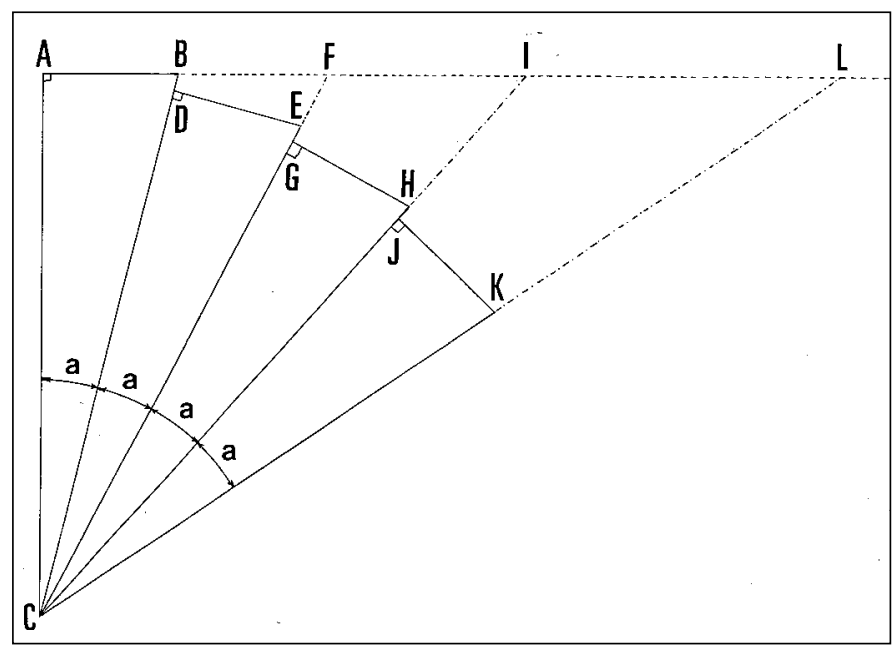

Figura 2. Relação entre ângulos e suas tangentes. (A proporção 100 $\overline{A B}=\overline{A C}$ não foi seguida)

\begin{tabular}{|c|c|c|c|c|c|}
\hline \multicolumn{3}{|c|}{ Múltiplos } & \multicolumn{3}{|c|}{ Submúltiplos } \\
\hline $10^{24}$ & yotta & Y & $10^{-24}$ & yocto & $\mathrm{y}$ \\
\hline $10^{21}$ & zetta & Z & $10^{-21}$ & zepto & z \\
\hline $10^{18}$ & exa & $\mathrm{E}$ & $10^{-18}$ & atto & a \\
\hline $10^{15}$ & peta & $P$ & $10^{-15}$ & femto & $f$ \\
\hline $10^{12}$ & tera & $\mathrm{T}$ & $10^{-12}$ & pico & $p$ \\
\hline $10^{9}$ & giga & $G$ & $10^{-9}$ & nano & $n$ \\
\hline $10^{6}$ & mega & M & $10^{-6}$ & micro & $\mu$ \\
\hline $10^{3}$ & quilo & k & $10^{-3}$ & mili & m \\
\hline $10^{2}$ & hecto & $\mathrm{h}$ & $10^{-2}$ & centi & c \\
\hline $10^{1}$ & deca & $d a$ & $10^{-1}$ & deci & d \\
\hline
\end{tabular}

rindo-lhe significados próprios a cada ordem de grandeza representada (Tabela 1 ).

Então, a distância $1,296.10^{16} \mathrm{~m}$ pode ser representada por $12,96.10^{15}$ ou 12,96 Pm (petâmetros), assim como a do comprimento de onda daquela radiação (vermelha), isto é, $7.10^{-7} \mathrm{~m}$ ser dada como $700.10^{-9} \mathrm{~m}$, o mesmo que $700 \mathrm{~nm}$ (nanômetros) ${ }^{(*)} \mathrm{ou}$, ainda, $0,7 \cdot 10^{-6} \mathrm{~m}$, igual a $0,7 \mu \mathrm{m}$ (micrômetros) ${ }^{(* *)}$. Da mesma forma, um quilômetro é igual a 1000 metros $\left(1 \mathrm{~km}=10^{3} \mathrm{~m}\right)^{(* * *)}$.

Diz-se, então, que a "ordem de grandeza" é dada pelo expoente (na base dez) mais próximo da quantidade medida; $0,7.10^{-6}$ está mais próxima de $10^{-6}$ do que de $10^{-7}(700 \mathrm{~nm}$ estão mais próximos de $1000 \mathrm{~nm}$ do que de $100 \mathrm{~nm}$; ou, equivalentemente: $0,7 \mu \mathrm{m}$ estão mais próximos de $1 \mathrm{~m} \mu$ do que de $0,1 \mathrm{~m} \mu)$; logo $-6\left(\right.$ ou $\left.10^{-6} \mathrm{~m}\right)$ é a ordem de grandeza do referido comprimento de onda, enquanto 16 (ou $10^{16} \mathrm{~m}$ ) é a ordem de grandeza da distância percorrida pela luz em 500 dias.

Isso é importante, porque a escala em que se faz a medida deve estar relacionada à sua ordem de grandeza. Para medir a altura de uma pessoa, a escala deve ser da ordem de grandeza de $10^{0} \mathrm{~m}$.

\section{E) Escalas, sensibilidades, leituras e interpolações}

Medidas devem ser feitas por instrumentos, ou métodos, cujas leituras, ou resultados, possam reproduzir múltiplos, ou submúltiplos, da unidade requerida. A escala desse instrumento, ou método, é, então, algo fundamentalmente adaptável à própria medida. Por exemplo, para avaliação da massa de um navio não se pode usar qualquer instrumento com escala da ordem de grandeza de $100 \mathrm{~kg}$, com sensibilidade de um quilo-

\footnotetext{
${ }^{(*)}$ : O nm (nanômetro), ou bilionésimo do metro, é o símbolo (e o nome) moderno para substituir $\mathrm{m} \mu$ (milimicron; no plural, milimicra). Ainda, $1 \mathrm{~nm}=10 \mathrm{~A}$ (Angstrons).

${ }^{(* *)}$ : O $\mu \mathrm{m}$ (micrômetro), ou milionésimo do metro, é o símbolo (e o nome) moderno para substituir o $\mu$ (micron; no plural, micra).

${ }^{(* * *)}$ : Quilograma tem por símbolo kg (e não $\mathrm{Kg}$ ), já que essa quantidade (e não a do grama) é a que representa a unidade de massa no S.I. Mas para o milésimo do quilograma diz-se "grama", um nome já consagrado pelo uso e que se conserva, por razões históricas, como "unidade". Não se diz, então, miliquilograma, assim como $10^{-6} \mathrm{~kg}=10^{-3} \mathrm{~g}$ é miligrama (=1 mg) e não o microquilograma $(1 \mu \mathrm{kg})$.
} 
grama, a qual serviria para avaliar a massa ("peso") de pessoas adultas mas, também, não se aplicaria à medição da de moedas.

Assim, além da ordem de grandeza, a escala deve apresentar divisões que permitam a leitura de suas frações. Dependendo de como essas divisões são apresentadas, uma leitura mais ou menos apurada dessas frações poderá ser feita. É isso que significa a sensibilidade do instrumento. Por exemplo, há balanças cujas divisões são de 100 em 100 g. Uma leitura poderá então ser feita (geralmente por aproximação) como $8100 \mathrm{~g}$, ou $8200 \mathrm{~g}$, ou ainda (quando não coincida com uma dessas divisões e fique entre elas), como $8150 \mathrm{~g}$. A esta se diz ser uma avaliação por interpolação. Obviamente, essa interpolação não deve exprimir uma informação ainda mais detalhada, como $8145 \mathrm{~g}$, pois isso requereria que a escala se apresentasse com sensibilidade (intervalos) de $5 \mathrm{~g}$ ou, no máximo, $10 \mathrm{~g}$ (para a leitura de interpolação entre $8140 \mathrm{~g} \mathrm{e} 8150 \mathrm{~g}$ ).

É muito comum que sejam apresentadas, em instrumentos eletrônicos, leituras automáticas, certamente também sujeitas a um limite. Por exemplo, numa balança que forneça leitura em quilogramas, mas com a informação de um algarismo depois da vírgula (isto é, com sensibilidade de $0,1 \mathrm{~kg}$ ), as leituras de $8,1 \mathrm{~kg}$ ou $8,2 \mathrm{~kg}$ serão possíveis alternativas para uma medida. Medições abaixo de $8150 \mathrm{~g}$ serão dadas como $8,1 \mathrm{~kg}$, assim como as acima desse valor aparecerão como $8,2 \mathrm{~kg}$. O instrumento, simplesmente, não chega à informação de uma leitura mais refinada, mas a de interpolação poderá até ser referida, quando o mostrador estiver alternando os valores 8,1 e 8,2. Por outro lado, a sensibilidade instrumental não é sinônima de sua qualidade ou capacidade discriminativa da medida, isto é, da exatidão e precisão com que elas possam ser feitas; mas, tão somente, refere-se ao interesse da informação. Para medir a massa de uma pessoa basta, freqüentemente, a informação sobre ela em centigramas, deixando de haver interesse por expressões que cheguem a miligramas, ainda que a balança seja adequada para tal.

De qualquer modo, como reagirá o mostrador a uma medida precisa de $8150 \mathrm{~g}$ ? Ou, equivalentemente, como classificar uma leitura de 173,5 cm se os requerimentos de estudo exijam que todas apareçam em números inteiros dessa subunidade (173 ou 174)? A resposta é arbitrária: o valor (aproximado para menos, ou para mais) será estabelecido por uma convenção, uma programação do instrumento; por exemplo: em caso da medida coincidentemente na metade entre as que possivelmente interessam, a aproximação será dada para a de algarismo maior. Obviamente a regra seguirá para todas as demais condições similares.

\section{F) Algarismos significativos}

Numa medida, a quantidade de algarismos dependerá da escala e refletirá a significância de sua exatidão ou precisão (ver a seguir) Por exemplo, a altura de uma pessoa poderá ser referida como $1,74 \mathrm{~m}$ o que se traduz, igualmente, por $174 \mathrm{~cm}$ (três algarismos significativos). Mas se for apresentada como 1,740 m (quatro algarismos significativos), a interpretação

\begin{tabular}{|cccc|}
\hline $\begin{array}{c}\text { Tabela 2. Expressão numérica e significados de uma medida } \\
\text { Expressão de }\end{array}$ & $\begin{array}{c}\text { Significado da } \\
\text { medida }\end{array}$ & $\begin{array}{c}\text { Unidades } \\
\text { significativas } \\
\text { (metros) }\end{array}$ \\
32 & $\geq 31,5$ & e $<32,5$ & metros \\
32,0 & $\geq 31,95$ & e $<32,05$ & decímetros \\
32,00 & $\geq 31,995$ & e $<32,005$ & centímetros \\
32,000 & $\geq 31,9995$ & e $<32,0005$ & milímetros \\
\hline
\end{tabular}

será diferente: $1740 \mathrm{~mm}$, isto é: igual a, ou maior que $1739,5 \mathrm{~mm}$ e menor que $1740,5 \mathrm{~mm}$. A informação do terceiro algarismo (neste caso, incidentalmente o zero, mas poderia ser 3 ou 8) dá a entender que a acurácia ou a sensibilidade com que a medida é feita chega à casa dos milímetros. Ao contrário, dizendo-se $174 \mathrm{~cm}$, o significado é o de que não se trata de $175 \mathrm{~cm}$ (mas poderia ser 174,4 cm) ou $173 \mathrm{~cm}$ (mas poderia ser $173,6 \mathrm{~cm})$. A Tabela II ilustra bem a relação entre a expressão numérica e o significado de uma medida. Em suma, algarismos significativos são aqueles sobre os quais a informação não deixa dúvidas.

Em cálculos, a expressão do resultado fica também condicionada ao número de algarismos significativos das parcelas de uma soma ou subtração; ou dos fatores de um produto, ou qüociente. A soma (ou subtração) de parcelas nunca pode ter mais algarismos significativos do que os da parcela que os contenha menos. Em outras palavras, o resultado de uma soma não pode ter maior sensibilidade do que a menos sensível de suas parcelas. Na operação de adição das parcelas: $6 \mathrm{~kg}+$ $317 \mathrm{mg}(=0,000317 \mathrm{~kg})+40 \mathrm{~g}(=0,040 \mathrm{~kg})$, ou seja, com um, três e dois algarismos significativos, a resposta aritmética $(6,040317 \mathrm{~kg})$ é errada, prevalecendo como resposta o valor que tem um algarismo significativo, isto é, $6 \mathrm{~kg}$. Na verdade, ao estabelecer o valor da primeira parcela como $6 \mathrm{~kg}$, a medida significa que ela não é $7 \mathrm{~kg}$ (mas poderia ser 6,499 kg) e não é $5 \mathrm{~kg}$ (mas poderia ser $5,501 \mathrm{~kg}$ ). Ora, a possível variação é da ordem de $1 \mathrm{~kg}$, aproximadamente, não sendo correto admitir-se que com tal inexatidão a soma seja afetada por parcelas com valor em gramas ou miligramas. Na prática, é o mesmo que considerar que o registro do peso de uma pessoa numa balança, dado como $80,4 \mathrm{~kg}$, possa se alterar por ela colocar duas moedas num bolso e inspirar fundamente...

Em multiplicações, também: o produto não pode ter mais algarismos significativos do que os do fator com menor número deles. Assim, o espaço percorrido em 20 segundos, com uma velocidade de $100 \mathrm{~km} /$ hora será $20 \mathrm{~s} .100 \mathrm{~km} / 3600 \mathrm{~s}$ deve ser dado não pela dízima $0,555 \ldots \mathrm{km}$, nem pela aproximação $556 \mathrm{~m}$ ou, menos ainda, $555,556 \mathrm{~m}$, mas por $0,56 \mathrm{~km}^{(*)}$ ou $56.10^{1} \mathrm{~m}$ (diferente de $560 \mathrm{~m}$ ). Aumentar a precisão da medida da velocidade (100.000 m/hora) não mudará a expressão do

\footnotetext{
(*): Zeros à esquerda do primeiro algarismo (diferente dele) não são significativos. Assim, $12 \mathrm{~mm}=0,012 \mathrm{~m}=0,000012 \mathrm{~km}$ são expressões com apenas dois algarismos significativos. Em todos os demais casos, o zero é contado como algarismo significativo.
} 
resultado; mas se aprimorar a do tempo, afirmando-se que é 20,00 segundos (4 algarismos significativos), então: 20,00 s x $100 \mathrm{~km} / 3600 \mathrm{~s}=0,556 \mathrm{~km}=556 \mathrm{~m}$ (três algarismos significativos, por causa da medida da velocidade, com apenas essa quantidade de significância), mas não 555,6 m.

\section{G) Precisão e Exatidão}

Embora esses dois termos sejam considerados costumeiramente como sinônimos, possuem diferentes significados quando o assunto é a confiabilidade da quantificação.

Precisão (de um instrumento ou método) refere-se à invariabilidade com que são obtidos os valores de medidas realizadas em condições rigorosamente similares. Uma balança que registre sempre o peso de um objeto como 936 gf é tida como precisa. $\mathrm{Na}$ verdade ela pode estar descalibrada (o peso a ser medido seria, na realidade, $1000 \mathrm{gf}$ ), mostrando o valor com inexatidão, com um erro sistemático. Um exemplo corriqueiro é o dos relógios: a quase totalidade deles trabalha num ritmo constante, preciso; mas alguns podem ser inexatos, atrasando-se ou adiantando-se (sistematicamente). A precisão é importante porque se conhecendo a eventual inexatidão do instrumento (por exemplo, no caso do relógio, um atraso cumulativo de um minuto a cada hora) saber-se-á que a marcação 18 horas e 41 minutos (no primeiro dia de trabalho do relógio, a partir de 0 h) significará, exatamente, 19 horas; e que idêntico horário do dia seguinte $(19 \mathrm{~h})$ corresponderá a 18 horas e 17 minutos nesse relógio inexato, mas preciso! O erro sistemático foi aqui mostrado como aditivo, mas pode ser relativo; isto é, o valor apresentado pelo instrumento aparecer com uma leitura de $\mathrm{x} \%$ a mais (ou a menos) referentemente à exata.

Exatidão, por outro lado, pressupõe a variabilidade das medidas (embora feitas em condições idênticas), sendo o valor central da distribuição (geralmente a média aritmética) o "exato". Portanto, quanto maior a quantidade de medidas feitas, mais exata será sua representação. Uma balança pode ser imprecisa, mas sendo exata referirá o valor real da medida, ainda que em nenhuma delas a leitura se apresente. Por exemplo, um peso de 1000 gf, registrado com valores 980, 1008, 1003, 1014, $995(\mathrm{~m}=1000)$. Um dado sobre a inexatidão do instrumento ou método é então diretamente fornecido pela variabilidade de suas medidas. No caso acima, $s=13,17$. Exatidão total há quando $\mathrm{s}=0$.

Obviamente, é recomendável que todo instrumento ou método possua precisão e exatidão. A primeira dessas qualidades de fidedignidade é controlada pela calibração, feita por comparação à medida de um padrão cujo valor (preciso) é conhecido. Sem esse conhecimento, o desvio da escala não pode ser aferido. Já a segunda característica (exatidão) pode ser conseguida pelo aumento infinito do número de medidas. Ou, pelo menos, com um número finito, mas até a aproximação desejada ou necessária.

\section{H) Fidedignidade, significância clínica e estatística}

Há grande diferença entre o que se pode ter como signifi- cado fidedigno (exato, preciso) de uma medida e seu valor prático. Por exemplo, admita-se que, entre três ceratômetros, um forneça a medida de curvatura corneal com significado de um dígito seqüencial ao de milímetros (de raio de curvatura), outro de dois e outro de três.

A medida com três dígitos (chegando a milésimos de milímetro, portanto da ordem de grandeza métrica -6 , isto é, um micrômetro) é de uma ordem de grandeza "maior" (em termos absolutos) que a do tamanho de células epiteliais da córnea $\left(10 \mu \mathrm{m}=10^{-5} \mathrm{~m}\right)$. Além de tanta acurácia sobrepassar a realidade anatômica (célula epitelial) da estrutura sobre a qual se faz a medida, a relação entre raio de curvatura (r, mm) e valor dióptrico da córnea (D) é geralmente dada por $\mathrm{k}=\mathrm{rD} \simeq 336 \simeq$ 1000/3. Daí, as equivalências das variações de medidas decimilimétricas $(0,100 \mathrm{~mm})$, centimilimétricas $(0,010 \mathrm{~mm})$ e micrométrica $(1 \mu \mathrm{m})$ serem, respectivamente (para valores de raio de curvatura acima dos do padrão $7,800 \mathrm{~mm})^{(*)}$ :

$7,900 \mathrm{~mm}(=42,19 \mathrm{D})-7,800 \mathrm{~mm}(=42,74 \mathrm{D})=0,100 \mathrm{~mm}(=-0,55 \mathrm{D})$ $7,810 \mathrm{~mm}(=42,68 \mathrm{D})-7,800 \mathrm{~mm}(=42,74 \mathrm{D})=0,010 \mathrm{~mm}(=-0,06 \mathrm{D})$ $7,801 \mathrm{~mm}(=42,73 \mathrm{D})-7,800 \mathrm{~mm}(=42,74 \mathrm{D})=0,001 \mathrm{~mm}(=-0,01 \mathrm{D})$

Note-se que se a sensibilidade do instrumento fosse limitada aos valores $10^{-1} \mathrm{~mm}, 10^{-2} \mathrm{~mm}$ e $10^{-3} \mathrm{~mm}$, respectivamente, os resultados das operações, em função do número de algarismos significativos nos respectivos casos (dois, três e quatro) seriam:

$7,9 \mathrm{~mm} \quad(=42 \mathrm{D})-7,8 \mathrm{~mm} \quad(=43 \mathrm{D})=0,1 \mathrm{~mm} \quad(=-1 \mathrm{D})$
$7,81 \mathrm{~mm} \quad(=42,7 \mathrm{D})-7,80 \mathrm{~mm} \quad(=42,7 \mathrm{D})=0,01 \mathrm{~mm} \quad(=0 \mathrm{D})$
$7,801 \mathrm{~mm} \quad(=42,73 \mathrm{D})-7,800 \mathrm{~mm} \quad(=42,74 \mathrm{D})=0,001 \mathrm{~mm} \quad(=-0,01 \mathrm{D})$

De qualquer modo, o instrumento cuja sensibilidade pertença à ordem de grandeza métrica - 4 (decimilimétrica) trabalha com unidades inteiras de dioptrias ópticas, sendo pois inadequado a usos clínicos (geralmente as lentes convencionais consideram variações de $0,25 \mathrm{D}$ e, raras vezes, a metade disso). Mas o de ordem de grandeza métrica -6 (micrométrica) chega ao detalhamento de centésimos de dioptria, obviamente exagerado, pelo menos para a prática clínica convencional.

Do ponto de vista estatístico, supondo-se que as médias de duas distribuições de medidas ceratométricas tenham variado por $0,2 \mathrm{D}$, que esse resultado seja estatisticamente significativo, mas que os valores resultem de operações aritméticas obtidas por leituras cuja sensibilidade é da ordem de $0,1 \mathrm{~mm}$ no raio de curvatura, a diferença entre as duas coleções de dados não pode ser cientificamente confirmada. E mesmo que a sensibilidade instrumental fosse de $0,001 \mathrm{~mm}$, a equivalência de cada intervalo dióptrico entre as respectivas leituras métricas (isto é, em torno de $0,55 \mathrm{D}$ ) encontra-se superior à diferença estatística encontrada, o que também põe em dúvida a validade das conclusões.

Por fim, suponha-se que tenha sido demonstrado um resultado estatístico significativo, com base em medidas de alta sensibilidade (com as quais aqueles se tornam "confiáveis"). Mesmo então, o estudo pode não ter significância clínica. Por

\footnotetext{
(*): A significância de quatro algarismos no resultado é possível se for usada a expressão $\mathrm{r} \mathrm{D} \simeq 1000 / 3$. Mas se $\mathrm{r} . \mathrm{D} \simeq 336$, os três algarismos dessa constante limitarão, também, o resultado.
} 
exemplo, suponha-se que um tipo de cirurgia aplicada a um grupo de endotrópicos com desvio $+50^{\Delta} \pm 2^{\Delta}$ tenha reduzido o estrabismo a valores $+38^{\Delta} \pm 1^{\Delta}$. Mesmo sem cálculos mais complicados pode-se notar que a quase totalidade da distribuição de valores (três desvios-padrão para mais ou para menos, cerca de $99 \%$ ) fica entre $+56^{\Delta} \mathrm{e}+44^{\Delta}$ antes da operação, contra $+41^{\Delta} \mathrm{e}+35^{\Delta}$ depois dela. Duas distribuições bem discrepantes: pode-se concluir que a cirurgia dá um resultado estatisticamente significativo na correção (absoluta) do ângulo do estrabismo. Mas que é (relativamente) insuficiente para que ela seja aceita como boa prática clínica.

Por outro lado, "100\% de melhora" a partir de uma acuidade visual 0,1 será geralmente, um resultado ainda insatisfatório como objetivo de muitos dos procedimentos em Oftalmologia. Assim, a pertinência da escolha de um parâmetro absoluto ou relativo para medir um resultado, assim como a do significado médico (clínico ou cirúrgico) de um tratamento para obtê-lo, deve ser cuidadosamente considerada em cada condição específica.

ABS TRACT

The nature of mensurations, terminologies and the importance of their uses are commented regarding numerical systems, logarithmic representations, units (with applications to measurements of the index of refraction, visual acuity and angles), orders of magnitude, scales, sensitivity, instrumental readings and interpolations, significant digits, precision and accuracy, reliability and significance (clinical and statistical).

Keywords: Visual acuity; Refraction, ocular; Measures; Mathematics; Visual tests/instrumentation; Reproducibility of results; Models, theoretical

\section{Congresso Internacional de Catarata e Cirurgia Refrativa}

\section{Centro de Convenções de Pernambuco}

\section{1 a 24 de abril de 2.004 OLINDA - PE}

INFORMAÇÕES: Tel.: (81) 3442-1940 - Tel./Fax: (81) 3265-7419

E-mail: catarata@hotlink.com.br

Home Page: www.catarata-refrativa.com.br 